Introduction Numerous studies have questioned the value of taking biopsies in endoscopic macroscopically normal mucosa. Inappropriate biopsies increase financial and time strains on NHS endoscopy and pathology services. Taking a biopsy adds to each procedure a cost of $£ 56$ for gastroscopy and $£ 66$ for colonoscopy. With $4.5 \%$ yearly increase in demand for histopathology services and only $1.2-3 \%$ per year increase recruitment of cellular pathologists, number of patients waiting longer than 6 weeks for diagnostic results has increased by $17 \%$ per year since 2010-11.

Methods We did a retrospective study of diagnostic gastroscopies and colonoscopies performed across 3 district-general hospitals (January-November 2018) in United Lincolnshire Hospitals NHS Trust. Endoscopic reports were examined for age, sex, indication, endoscopic diagnosis, biopsies taken (yes/ no), and operator (gastroenterologist/surgeon/nurse endoscopists $\{\mathrm{NE}\})$. We classified 'biopsy not indicated' when mucosa was described as 'normal' for indications of anaemia, rectal bleeding, weight-loss in colonoscopies, and dyspepsia, vomiting and abdominal pain in gastroscopies.

Results A total of 326 gastroscopies and 355 colonoscopies were included. 170 procedures fulfilled the 'biopsy not indicated' criterion, of which 59\% had multiple biopsies (at least 5 specimens) taken. Biopsy rates among gastroenterologists, surgeons, and NEs in 'biopsy not indicated' were 53\% (39/ 73), 60\% (42/70) and 70\% (19/27) respectively. Using Chisquare, there was no statistically significant difference between various groups: surgeons and NEs $(p=0.34)$, surgeons and gastroenterologists $(\mathrm{p}=0.42)$, and gastroenterologists and NEs $(\mathrm{p}=0.12)$.

Conclusions Our study showed that a significant number of biopsies are performed without good indication. Further, no significant inter-operator variability was seen. Both British Society of Gastroenterology and National Institute for Health and Care Excellence have published guidance on when biopsy is indicated, but there are few high-level recommendations on when not to biopsy. Taking a biopsy cost of $£ 103$ a potential of $£ 10330$ could have been saved during our study period. Findings from our study mandate development of such guidance, followed by training of all endoscopists and UK-wide audit of local practice to ensure compliance with guidelines. The implementation of such strategies has been proven effective at a local level, and if adopted nationally can significantly optimise financial burden on the NHS and reduce waiting times.

\section{P354 A MULTI-CENTRE AUDIT OF LOWER GASTROINTESTINAL BLEEDING MANAGEMENT}

${ }^{1}$ Michael Davies*, ${ }^{2}$ Tristan Townsend, ${ }^{3}$ Violeta Razanskaite, ${ }^{4}$ James Morgan, ${ }^{5}$ Daniyal Baig, ${ }^{6}$ James Fox, ${ }^{6}$ Vinay Kumar, ${ }^{3}$ Nada Elamin, ${ }^{3}$ loannis Papamargaritis, ${ }^{1}$ Zehra Zaidi, ${ }^{1}$ Heitham Zeglam, ${ }^{2}$ Doug Penman, ${ }^{2}$ Thomas Conley, ${ }^{2}$ Joe Fiske, ${ }^{4}$ Kieran Walker, ${ }^{2}$ Khoon Kok, ${ }^{2}$ Mira Swaminathan, ${ }^{2}$ Sreedhar Subramanian, ${ }^{2}$ Phil Smith. ${ }^{1}$ Countess of Chester Hospital, Chester, UK; ${ }^{2}$ Royal Liverpool University Hospital, Liverpool, UK; ${ }^{3}$ Aintree University Hospital, Liverpool, UK; ${ }^{4}$ Arrowe Park Hospital, Wirral, UK; ${ }^{5}$ Whiston Hospital, Merseyside, UK; ${ }^{6}$ Macclesfield District General Hospital, Macclesfield, UK

\subsection{6/gutjnl-2020-bsgcampus.428}

Introduction Two national reviews identified unwanted variation in the care of patients with gastrointestinal haemorrhage. British Society of Gastroenterology (BSG) acute lower gastrointestinal bleeding (LGIB) guidelines define a specific management approach including risk stratification, investigation and intervention for patients presenting with LGIB. Guideline adoption, impact on 'real-world' clinical outcomes and resource implications are not known. The Mersey Gastroenterology Network conducted a region-wide, multi-centre, pilot audit of LGIB management in relation to BSG guidance to assess this.

Methods Patients aged $\geq 16$ years presenting with LGIB to five hospital trusts from June 1st-July 1st 2019 were included. Data on presentation, management and outcomes of patients were recorded. These were audited against BSG guidelines.

Results 104 patients were included. Mean age at presentation was 60 years (range 23-96). A Shock Index (SI) >1 was rare $(7.7 \%)$, with a mean SI of 0.69 on presentation. $67 \%$ of patients haemodynamically unstable despite initial resuscitation underwent computed tomogram angiography (CTA). 21 patients (20.2\%) had a low risk Oakland Score (OS) ( $\leq 8$ points); 29\% were admitted whilst $71 \%$ were discharged for outpatient management. 75 patients $(78.9 \%)$ had a high-risk OS (>8 points); 61\% were admitted and $39 \%$ were discharged. In patients discharged with a highrisk OS (median 11), 6.9\% were readmitted within 30 days. Admitted patients underwent CTA (10.2\%), colonoscopy (8.5\%), flexible sigmoidoscopy $(3.4 \%)$ or OGD $(22 \%)$ as initial investigation. $55.9 \%$ were observed without diagnostic investigations for an average of 3 days before discharge. Rebleeding within 7 days, readmission within 30 days and inpatient mortality was $9.3 \%, 15.8 \%$ and $3.4 \%$ respectively. The median length of admission was 3 days (range 0-60). $38.5 \%$ of patients were managed in line with BSG guidance. The most common deviation was patients with an OS $>8$ being monitored without investigation during admission (31.7\%).

Conclusions LGIB management does not follow current guidelines. We report low mortality and readmission rates, even in patients discharged with a 'high-risk' OS. Admitted patients rarely receive an urgent inpatient colonoscopy. We aim to collect data over an extended period to provide further insights and analyse economic and resource feasibility of guideline adherence.

\section{P355 DESIGN AND IMPLEMENTATION OF THE FIRST ONE- STOP MULTIDISCIPLINARY CLINIC FOR LYNCH SYNDROME IN THE UK}

Bianca DeSouza*, Vishakha Tripathi, Adam Shaw, Sabina Demartino, Giovanni Tritto, Mark George, Alexis Schizas, Gautam Mehra, Clare Firth, Louise Izatt, Anju Kulkarni. Guy's and St Thomas' NHS Foundation Trust, London, UK

\subsection{6/gutjnl-2020-bsgcampus.429}

Introduction The clinical benefit of a multidisciplinary clinic (MDC) model has been well documented for a variety of medical conditions. We designed and implemented a novel MDC for Lynch syndrome (LS) patients, which aims to improve treatment outcomes, participation in research trials, and patient satisfaction.

Methods From January 2019, LS patients in our region were invited to attend a bimonthly MDC offering gene-specific evidence-based cancer risk management. Patients could choose to see the gastroenterology, colorectal, gynaecology, medical oncology, research, and clinical psychology teams. Clinical outcomes and patient satisfaction were evaluated over 12 months. Results Thirty-eight LS patients were seen (8 MLH1, 18 MSH2, 8 MSH6, 4 PMS2); mean age was 46y (range: 20- 